\title{
Iranian brown propolis possesses neuroprotective effect against ischemic neuronal damage in mice
}

\author{
Gholamreza Bazmandegan $^{1,2}{ }^{\circledR}$, Ali Shamsizadeh ${ }^{1,3}$, Mohsen FathiNajafi ${ }^{4^{\circledR}}$, Zahra Assadollahi $^{{ }^{(}}$, Mohammad

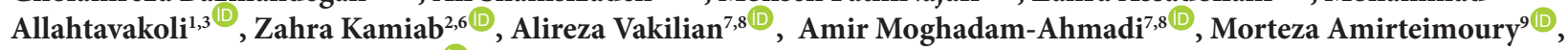 \\ Mohammad Taher Boroushaki ${ }^{10 *(\mathbb{D})}$ \\ ${ }^{1}$ Physiology-Pharmacology Research Center, Research Institute of Basic Medical Sciences, Rafsanjan University of Medical Sciences, Rafsanjan, Iran \\ ${ }^{2}$ Clinical Research Development Unit, Ali-Ibn Abi-Talib Hospital, Rafsanjan University of Medical Sciences, Rafsanjan, Iran \\ ${ }^{3}$ Department of Physiology and Pharmacology, School of Medicine, Rafsanjan University of Medical Sciences, Rafsanjan, Iran \\ ${ }^{4}$ Razi Vaccine and Serum Research Institute, Mashhad Branch, Agricultural Research, Education and Extension Organization (AREEO), Mashhad, Iran \\ ${ }^{5}$ Department of Epidemiology and Biostatistics, School of Medicine, Rafsanjan University of Medical Sciences, Rafsanjan, Iran \\ ${ }^{6}$ Department of Family Medicine, Ali-Ibn Abi-Talib Hospital, School of Medicine, Rafsanjan University of Medical Sciences, Rafsanjan, Iran \\ ${ }^{7}$ Non-Communicable Diseases Research Center, Rafsanjan University of Medical Sciences, Rafsanjan, Iran \\ ${ }^{8}$ Department of Neurology, Ali-Ibn Abi-Talib Hospital, School of Medicine, Rafsanjan University of Medical Sciences, Rafsanjan, Iran \\ ${ }^{9}$ Student Research Committee, Rafsanjan University of Medical Sciences, Rafsanjan, Iran \\ ${ }^{10}$ Pharmacological Research Center of Medicinal Plants, Faculty of Medicine, Mashhad University of Medical Sciences, Mashhad, Iran
}

\section{A R T I C L E I N F O}

\section{Article Type:}

Original Article

\section{Article History:}

Received: 20 May 2019

Accepted: 19 August 2019

\section{Keywords:}

Cerebral ischemia

Brown propolis

Neuroprotection

Polyphenol

\begin{abstract}
A B S T R A C T
Introduction: Stroke is one of the leading causes of death and disability worldwide. Propolis, a polyphenol-rich resinous product processed by honeybees from a variety of plant sources, has a set of biological activities. We investigated the neuroprotective effect of Iranian brown propolis (IBP) in a mouse model of permanent middle cerebral artery occlusion (MCAO).

Methods: Experimentally, water extracts of propolis (WEPs) were obtained from Kerman (KeWEP) and Khorasan Razavi (KhWEP) provinces, Iran. The chemical characterization and total polyphenol content of WEPs were determined using the Folin-Ciocalteu assay and gas chromatography-mass spectrometry (GC-MS). Animals were divided into eight experimental groups including: sham, control, and three groups each of which KeWEP- and KhWEP-treated mice. The drugs were administered at doses of 30, 100 and $200 \mathrm{mg} / \mathrm{kg}$, intraperitoneally (IP), during four different time points. Infarct volume and brain edema were measured at $48 \mathrm{~h}$. Behavioral tests were evaluated at 4, 24 and 48-hour post stroke.

Results: The total polyphenol content was 1100 and $1400 \mathrm{mg} / \mathrm{L}$ in KhWEP and KeWEP respectively. Compared to the control group, the doses of 100 and $200 \mathrm{mg} / \mathrm{kg}$ in both samples decreased infarct volume. Brain edema was also reduced in all treatment groups. The dose of $200 \mathrm{mg} / \mathrm{kg}$ in both samples and $100 \mathrm{mg} / \mathrm{kg}$ in the KeWEP-treated group significantly increased grasping ability. Sensory-motor function was improved in all groups, too.

Conclusion: These results suggest that IBP may reduce ischemic brain injury by its neuroprotective effect on focal cerebral ischemia.
\end{abstract}

Implication for health policy/practice/research/medical education:

This paper provides pharmacological evidence for neuroprotective effect of Iranian brown propolis in a mouse model of permanent middle cerebral artery occlusion.

Please cite this paper as: Bazmandegan G, Shamsizadeh A, FathiNajafi M, Assadollahi Z, Allahtavakoli M, Vakilian A, et al. Iranian brown propolis possesses neuroprotective effect against ischemic neuronal damage in mice. J Herbmed Pharmacol. 2020;9(2):121-129. doi: 10.34172/jhp.2020.16.

\section{Introduction}

Stroke is the second leading cause of mortality and the most frequent cause of morbidity worldwide $(1,2)$. Ischemic brain injury, caused by a restricted perfusion, is accompanied by several signs and symptoms such as infarction, brain edema, neuroinflammation, oxidative stress and neurological disorders (3). During stroke, damage to the ischemic core causes cell death and 
growth of the infarction in the 'penumbra' (4). Though the core of the ischemic damage is not recoverable, the penumbra remains a target for the progress of therapeutic approaches (5).

A complex of pathophysiological mechanisms such as oxidative stress, inflammation, peri-infarct depolarization, excitotoxicity, and apoptosis precipitate neuronal progressive cell death in the penumbra (4). Currently, bating thrombolytic therapy, neuroprotective agents are under development for therapeutic purposes (6).

Propolis, a natural hive product, is a polyphenolrich resinous compound. Honeybees process it through blending the resinous sap acquired from the bark and sprouts of varied plants with own secretion $(7,8)$. It has been shown that the compounds and properties of propolis may depend on the vegetation, time and the region of gathering (9). More than 150 chemical compounds in propolis have been reported, mainly polyphenol category, some of which are as follows: fatty and phenolic acids and esters, flavonoids, pinocembrin, caffeic acid phenethyl ester, aromatic aldehydes and alcohols (10). Propolis has been known as a traditional medicine employed by human since the ancient period for its pharmaceutical properties (11). Indeed, various studies have demonstrated a wide range of biological properties such as antimicrobial (12), anti-apoptotic (13), antineuroinflammation (14), antioxidant (15) and neuroprotective effects (16). It has been demonstrated that Brazilian and Turkish propolis decreases neuroinflammation and neuronal damage following ischemic stroke (17-19). Phenolic compounds may act as the major components of the complicated composition and molecular diversity of propolis (12). These compounds are responsible for many biological properties and provide a protective effect in many pathological conditions $(12,20)$. Some compounds and pharmacological properties of Iranian brown propolis (IBP) have been revealed such as in-vitro antimicrobial and antioxidant activities (21-23). However, there is no report presenting evidence for the neuroprotective in-vivo activity of IBP. This study was conducted to assess the total polyphenol content of IBP and neuroprotective effect of its water extracts (WEPs) in a mouse model of permanent middle cerebral artery occlusion (MCAO).

\section{Materials and Methods}

Drugs and chemicals

Drugs and sources were as follows: 2,3,5-triphenyltetrazolium chloride (TTC) was purchased from Merck KGOA (Darmstadt, Germany). Ketamine hydrochloride, xylazine, diethyl ether and formaldehyde were purchased from Sigma-Aldrich.

Preparation of water extracts of propolis

Propolis was collected from beehives at the village Lalehzar in Kerman province and from another region (Hezar
Masjed) in Khorasan Razavi, Iran, and then was sent to Soren Tech Toos, Inc. Ltd. (Mashhad, Iran). Extraction of samples and assessing their total polyphenol content, using the Folin-Ciocalteu colorimetric assay with minor modifications, was performed by a company-specific protocol that was previously reported (21). The propolis was extracted with most water-soluble compounds. The quantity of total phenolic content was expressed as milligrams per liter (gallic acid standard) $(21,24)$.

Gas chromatography-mass spectrometry (GC-MS) The GC-MS analysis was executed with an Agilent Gas Chromatograph 6890 plus Agilent 5973N mass spectrometer instrument equipped with a $30 \mathrm{~m}$ long, 0.25 $\mathrm{mm}$ id, $0.25 \mathrm{~mm}$ film thickness BPX5 capillary column. The temperature was set from 50 to $300^{\circ} \mathrm{C}$ at a rate of 3 $\mathrm{C} / \mathrm{min}$. Helium was used as a carrier gas, flow rate $1 \mathrm{~mL} /$ $\min$. The injection was performed in split mode at $220^{\circ} \mathrm{C}$, ionization voltage $70 \mathrm{eV}$. The reconnaissance was done using chemstation software.

\section{Animals and treatments}

Seventy-two adult male Balb/C mice (30-35 g) were used in this study. Animals were housed in a pathogenfree facility on a 12-hour light/dark schedule and with ad libitum access to food and water. After acclimatization, animals were randomly divided into eight groups $(n=9)$ as follows: sham (craniotomy without MCAO), control (ischemia, treated with vehicle), and six therapeutic groups in each of which the effect of water extracts of Iranian brown propolis (WEPs) was evaluated. The WEPs were obtained from Kerman (KeWEP), and Khorasan Razavi (KhWEP), provinces of Iran. The KhWEP, with the doses of 30, 100, and $200 \mathrm{mg} / \mathrm{kg}$ (KhWEP30, KhWEP100, KhWEP200), as well as the KeWEP, with the same doses (KeWEP30, KeWEP100, KeWEP200), were intraperitoneally administered at 48, 24, 1 hours before, and 4 hours after the induction of focal brain ischemia. The dose of extracts was selected based on a previous study (17) and our preliminary results. Each mouse was weighed at the start of the study and before being killed. All groups were evaluated for the four indices of stroke injury (weight loss, infarct volume, brain edema and behavioral tests).

Permanent middle cerebral artery occlusion

Animals were anesthetized by intraperitoneally injection of ketamine $(80 \mathrm{mg} / \mathrm{kg})$ and xylazine $(8 \mathrm{mg} / \mathrm{kg})$ combination. The core body temperature was monitored and kept at $37^{\circ} \mathrm{C}$ during the anesthesia using a rectal probe. Induction of MCAO was performed by permanent occluding the right middle cerebral artery (MCA) as previously reported $(8,25)$. In brief, a small skin incision between the lateral part of the orbit and the external auditory meatus was made. Then, the connective tissue and muscles 
were slowly pulled aside. A small hole, about $1 \mathrm{~mm}$ in diameter, was drilled directly over the proximal part of the MCA, the Dura mater was removed, and the MCA was permanently cauterized using a thermal coagulator. The temporal muscle and connective tissue replaced, and skin was sutured. Animals were recovered in a heated cage and returned to their individual cages. Sham-operated animals (surgical sham group) were subjected to the same procedure except for the cauterization of MCA.

Measurement of infarct volume and brain edema Infarct volume and brain edema were calculated as previously described (26). In brief, 48 hours after right MCAO, animals were decapitated under deep anesthesia induced by diethyl ether. Brains were then removed and sliced into five coronal sections of $1 \mathrm{~mm}$ thickness. The prepared sections were stained with $2 \%$ TTC for 30 minutes at $37^{\circ} \mathrm{C}$ followed by overnight immersion in $10 \%$ phosphate-buffered formalin solution. The infarcted tissue remained unstained (white) while normal tissue was stained (red). The infarct volume was demarcated and analyzed using a color flatbed scanner (Scan jet 5370C, Hewlett-Packard, Palo Alto, CA, USA) and image J software (NIH Image, version 1.61 Bethesda, Maryland, USA). Infarct areas of all sections were added to determine the total infarct area, which was multiplied by the thickness of the brain sections $(1 \mathrm{~mm})$ to obtain the infarct volume. In order to compensate for the effect of brain edema, the corrected infarct volume was determined as follows: corrected infarct volume $=$ measured infarct area $\times\{1$-[(ipsilateral hemisphere area - contralateral hemisphere area)/contra lateral hemisphere]\}. Brain edema was determined using the following formula: edema $=$ (volume of the left hemisphere - the volume of the right hemisphere) / volume of the right hemisphere. The infarct volume and brain edema were expressed as percentages.

\section{Behavioral tests}

Latency to touch the label on the contralateral forepaw (sticky test score) was performed to assess sensory deficits. All animals in these groups were trained 24 and $48 \mathrm{~h}$ before MCAO and baseline score of their sensorial functions recorded immediately before surgery and then re-evaluated 4, 24 and 48 hours after the stroke induction. To perform this test, a $6 \times 6 \mathrm{~mm}$ sticky label was attached to the contralateral forepaw and the mean duration of touching of the label was determined (27). The hanging wire test was used to evaluate the grasping ability and forelimb strength. In this experiment, animals were hanging from hands on a horizontal thin steel wire $(1 \mathrm{~mm})$ which was stretched between two points to a height of 50 $\mathrm{cm}$ of the pad for a maximum duration of 1 minute. The time(s) until the mice fell was recorded as a test score. This test was performed for each animal four times (before and at 4, 24 and 48 hours after induction of ischemia) (28).

\section{Statistical analysis}

Statistical analysis of the data was presented as mean \pm SEM. Behavioral tests were compared by two-way ANOVA and individual differences determined by LSD test. Infarct volume and brain edema were compared by one-way ANOVA followed by Tukey's test. A value of $P<$ 0.05 was considered significant.

\section{Results}

Total polyphenol content and GC-MS analysis of IBP The total polyphenol content was 1100 and $1400 \mathrm{mg} / \mathrm{L}$ for KhWEP and KeWEP, respectively (Table 1). The KeWEP presented a substantially higher concentration of polyphenol when compared to KhWEP. The variety of polyphenol types differed somewhat from one sample to another. Figure 1 shows the GC-MS chromatograms for KhWEP and KeWEP, respectively. As shown in Table 1, the great flavonoids found in the extract from the KhWEP include sterubin and pinobanksin. In contrast, the major flavonoids in the KeWEP were quercetin and naringenin. Although the derivatives of cinnamic acid and phenolic acid esters were similar in both samples.

Table 1. Total polyphenol content and major components of WEPs

\begin{tabular}{clc}
\hline Samples & $\begin{array}{l}\text { Polyphenol } \\
\text { content (mg/L) }\end{array}$ & Major components \\
\hline KhWEP & 1100 & $\begin{array}{c}3,7 \text { dihydroxy } 5 \text { methoxy flavanone, } \\
\text { pinobanksin 3 acetates, cinnamic acid, } \\
\text { benzoic acid, pinobanksin 3 butanoate, } \\
\text { butanedioic }\end{array}$ \\
KeWEP & 1400 & $\begin{array}{c}\text { Quercetin, benzoic acid, cinnamic acid, } \\
\text { butanedioic, naringenin, propanoic acid }\end{array}$
\end{tabular}

WEPs: Water extract of propolis; KhWEP: Propolis water extract of Khorasan Razavi province; KeWEP: propolis water extract of Kerman province.
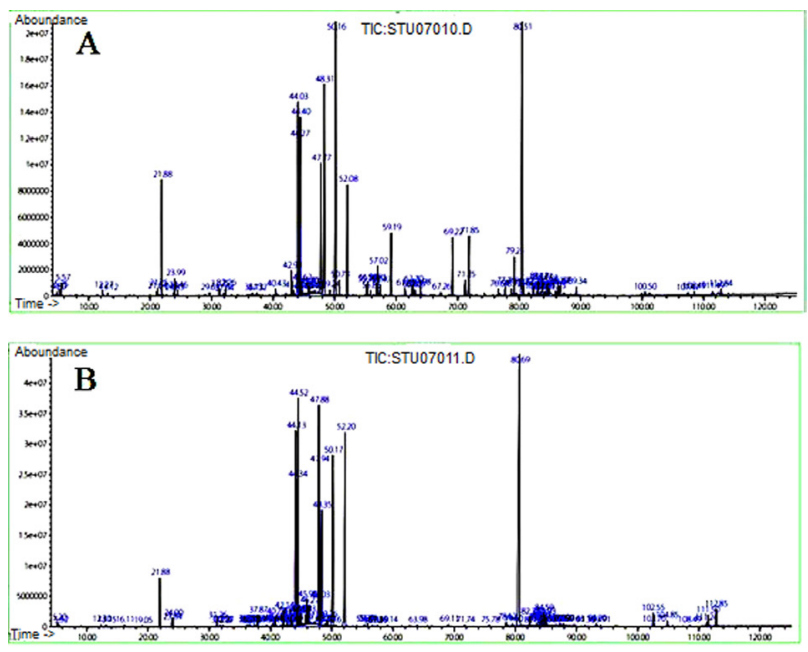

Figure 1. Gas chromatography-mass spectrometry of water extract of propolis from A: Khorasan Razavi province and B: Kerman province, Iran. 
Table 2. Initial and the final body weights in 8 experimental groups

\begin{tabular}{lccc}
\hline Groups & Initial BW & Final BW & BW loss \\
\hline Sham & $33.44 \pm 1.59$ & $33.00 \pm 1.44$ & $0.44 \pm .17$ \\
Control & $34.78 \pm 1.01$ & $30.78 \pm 1.07$ & $4.00 \pm 1.01 *$ \\
KhWEP 30 & $32.89 \pm 1.14$ & $29.89 \pm 1.16$ & $3.00 \pm 0.76$ \\
KeWEP 30 & $32.22 \pm 0.84$ & $29.13 \pm 0.71$ & $3.38 \pm 1.1$ \\
KhWEP 100 & $33.56 \pm 1.08$ & $30.00 \pm 1.34$ & $3.56 \pm 0.68$ \\
KeWEP 100 & $34.33 \pm 1.00$ & $32.56 \pm 1.33$ & $1.78 \pm 0.43$ \\
KhWEP 200 & $34.44 \pm 0.89$ & $31.67 \pm 1.40$ & $2.78 \pm 0.87$ \\
KeWEP 200 & $33.78 \pm 0.98$ & $32.33 \pm 0.88$ & $1.44 \pm 0.53$ \\
\hline
\end{tabular}

WEP: Water extract of propolis; KhWEP: Propolis water extract of Khorasan Razavi province; KeWEP: Propolis water extract of Kerman province.

Data are presented as the mean $\pm \operatorname{SEM}(n=9) .{ }^{*} P<0.05$ vs. sham group. Data were tested using the one-way ANOVA, Tukey post hoc test. $P<$ 0.05 was considered as statistically significant.

\section{Effect of IBP on body weight}

Table 2 presents results of initial and the final body weight also body weight loss. There was no significant difference between the weight of animals at the beginning or at the end of the experiments. Body weight loss, reduced in all treated groups compared to the control group. There was a significant weight loss in the control group compared to the sham group, but not a significant difference between the WEPs-treated groups with the sham group.

Effect of IBP on infarct volume

TTC staining of coronal brain sections and effect of WEPs on infarct volume are shown in Figure 2. No infarction was observed in the sham group. At the dose of $30 \mathrm{mg} / \mathrm{kg}$, no significant difference was observed between KeWEP $(14.56 \pm 1.6 \%)$ and KhWEP $(18.94 \pm 3.33 \%)$ compared to the control group $(17.75 \pm 1.32 \%),(P>0.05)$. There was a significant difference between the control group with

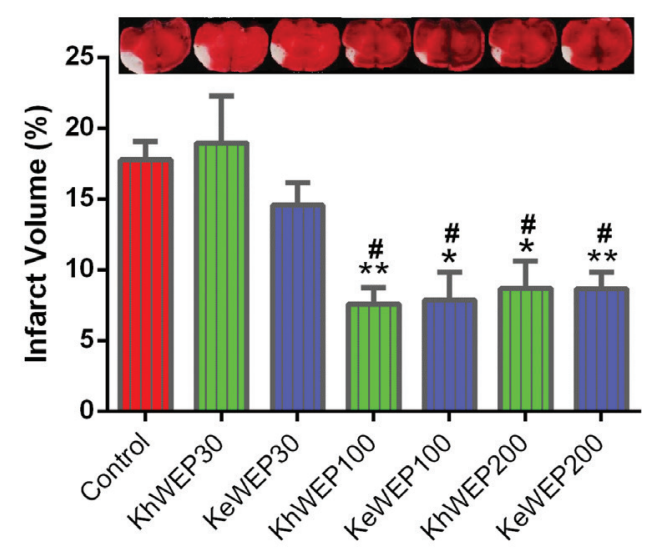

Figure 2. Effect of water extracts of IBP on infarct volume (\%). Data are presented as the mean \pm SEM $(n=9) .{ }^{*} P<0.001$ and ${ }^{*} P<0.01$ vs. control group, $\# P<0.01$ vs. dose 30 . Data were analyzed using the one-way ANOVA, Tukey post hoc test. $P<0.05$ was considered as statistically significant. IBP: Iranian brown propolis; KhWEP: Propolis water extract of Khorasan Razavi province; KeWEP: Propolis water extract of Kerman province.
KhWEP100 $(7.57 \pm 1.16 \%), \operatorname{KeWEP} 200(8.63 \pm 1.17 \%)$ $(P<0.001)$, also with KhWEP200 $(8.68 \pm 1.93 \%)$ and KeWEP100 $(7.85 \pm 1.95 \%)(P<0.01)$. Regardless of the area from which the substance was collected, there was a significant difference in infarct volume between both doses of 100 and 200, each compared to the dose of 30 $\mathrm{mg} / \mathrm{kg}(P<0.01)$. The difference between the effects of same doses from different regions was not statistically significant.

\section{Effect of IBP on brain edema}

Data analysis showed a significant difference between control $(10.18 \pm 2.02 \%)$ and surgical sham groups $(2.36$ $\pm 0.61 \%, P<0.01)$, which indicated considerable strokeinduced brain edema. All WEP-treated groups showed a significant difference compared to the control group. It was statistically significant for KhWEP30 (3.7 $\pm 0.82 \%)$, KeWEP30 (3.67 $\pm 0.93 \%)$, KhWEP100 (3.13 $\pm 0.66 \%)$ and KhWEP200 (3.45 $\pm 0.99 \%)$ at $P<0.01$ and for KeWEP100 $(4.18 \pm 0.71 \%)$ and KeWEP200 (4.36 $\pm 0.99 \%)$ at $P<0.05$ versus control group $(10.18 \pm 2.02 \%)$. Regardless of the drug dose, there was no significant difference between the samples of two regions in reducing brain swelling $(P>0.05)$. Similarly, regardless of WEPs preparation area, there was no significant difference between therapeutic doses of WEPs $(P>0.05)$. Similar doses of the two regions were not statistically significant $(P>0.05)$ (Figure 3 ).

Effect of IBP on hangingwire test

Hanging-wire results are shown in Table 3. Prior to the induction of ischemia, there was no significant difference between groups $(P>0.05)$. At different times after surgery (4, 24 \& 48 hours post-stroke), the animals showed significantly better performance when examined at 24 and 48 hours rather than 4 hours after stroke regardless of dosage and region $(P<0.001)$. There was no difference

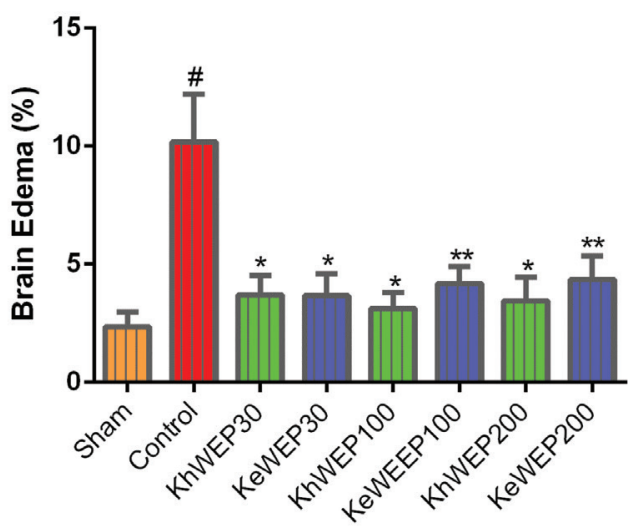

Figure 3. Effect of water extracts of IBP on brain edema (\%). Data are presented as the mean \pm SEM $(n=9)$. ${ }^{*} P<0.01 \&{ }^{* *} P<0.05$ vs. control group. Data were analyzed using the one-way ANOVA, Tukey post hoc test. $P<0.05$ was considered as statistically significant. IBP: Iranian brown propolis; KhWEP: Propolis water extract of Khorasan Razavi province; KeWEP: Propolis water extract of Kerman province. 
Table 3. Hang time remain $(\mathrm{Sec})$ on the thin wire in 8 experimental groups before and at 4,24 , and 48 hours after operation

\begin{tabular}{|c|c|c|c|c|}
\hline Groups & Before & After $4 \mathrm{~h}$ & After $24 \mathrm{~h}$ & After $48 \mathrm{~h}$ \\
\hline Sham & $41.56 \pm 3.64$ & $44.33 \pm 7.46$ & $47 \pm 6.49$ & $46.67 \pm 6.41$ \\
\hline Control group & $42.56 \pm 5.69$ & $11.44 \pm 3.42^{* *}$ & $16.22 \pm 4^{* *}$ & $17.44 \pm 6.52^{* *}$ \\
\hline KhWEP 30 & $41.11 \pm 5.63$ & $13.78 \pm 6.32$ & $23.22 \pm 5.28^{\#}$ & $25.78 \pm 8.81^{\#}$ \\
\hline KeWEP 30 & $42.11 \pm 4.27$ & $11.56 \pm 3.61$ & $20.44 \pm 7.04^{\#}$ & $20.22 \pm 6.65^{\#}$ \\
\hline KhWEP 100 & $42.22 \pm 2.73$ & $13.22 \pm 3.2$ & $25.89 \pm 6.9^{\#}$ & $26.22 \pm 5.78^{\#}$ \\
\hline KeWEP 100 & $42.33 \pm 6.22$ & $25.11 \pm 8.75^{*}$ & $37.56 \pm 7.92^{* \#}$ & $43.89 \pm 8.11^{* \#}$ \\
\hline KhWEP 200 & $41.56 \pm 5.1$ & $31.56 \pm 6.56 *+$ & $44 \pm 7.06^{* \#+}$ & $50.11 \pm 5.28^{* \#+}$ \\
\hline KeWEP 200 & $43.22 \pm 5.91$ & $25.78 \pm 7.42^{*+\dagger}$ & $38.11 \pm 7.82^{* \#+\dagger}$ & $45.33 \pm 6.35^{* \#+\dagger}$ \\
\hline
\end{tabular}

KhWEP: Propolis water extract of Khorasan Razavi province; KeWEP: Propolis water extract of Kerman province.

Data are presented as the mean \pm SEM $(n=9)$. Data were analyzed using the two-way ANOVA, LSD post hoc test. $P<0.05$ was considered as statistically significant.

$\# P<0.001$ vs. 4 h, ${ }^{* *} P<0.001$ vs. sham group, ${ }^{*} P<0.01$ vs. control group, $+P<0.05$ vs. KhWEP30 \& KhWEP100, $\uparrow+P<0.05$ vs. KeWEP30.

between 24 and 48 hours $(P>0.05)$. Irrespective of the time of evaluation (considering different regions and doses of drugs), hanging test results were statistically significant. There was a significant difference between control and surgical sham groups $(P<0.001)$; indicating deficits in endurance time following stroke induction. KeWEP at two doses (100 \& $200 \mathrm{mg} / \mathrm{kg}$ ) and KhWEP with a dose of $200 \mathrm{mg} / \mathrm{kg}$ significantly improved hanging scores compared to control group $(P<0.01)$. For the KhWEP, there was a significant difference between the dose of 200 and both doses of 30 and $100 \mathrm{mg} / \mathrm{kg}(P<0.05)$. However, in the case of KeWEP, such significant difference was only observed between the dose of 200 and $30 \mathrm{mg} / \mathrm{kg}(P<0.05)$. There was no significant difference between similar doses of both regions $(P>0.05)$.

Effect of IBP on the sticky test

Data analysis before the surgery displayed similar sensory performance in all groups $(P>0.05)$. At different times after surgery $(4,24$ or 48 hours post-stroke), sticky test results were significantly different at 48 hours compared to 4 and 24 hours $(P<0.001)$, regardless of region and dosage of WEPs. However, such significant difference was not observed between 24 and 4 hours intervals $(P>0.05)$.
Regardless of time, there was a significant difference in sticky test score concerning region and dosage of WEPs $(P<0.001)$. The difference between control and surgical sham groups was significant $(P<0.001)$, which indicates the stroke-induced sensory deficits. KeWEP and KhWEP at all doses demonstrated a significant difference compared to the control group $(P<0.05)$. The best therapeutic effects were observed at KhWEP200, so that made a significant difference not only compared to the control group but also with other WEP-treated groups $(P<0.05)$. There was no significant difference between similar doses of 30 and 100 $\mathrm{mg} / \mathrm{kg}$ in 2 regions $(P>0.05)$ (Table 4$)$.

\section{Discussion}

Using the neuroprotective agents in reducing neuronal damage and saving the penumbra cell is one of the main clinical goals of neuroscience research (29). Propolis, processed by the honey bees, is a natural polyphenol-rich compound with the widespread use in traditional and alternative medicine (20). Findings of the present study demonstrated that treatment with WEPs of IBP from two regions (KeWEP and KhWEP) provides neuroprotection via a significant reduction in stroke complications after permanent MCAO in mice. The IBP administration

Table 4. Touching time (s) on the sticky test in eight experimental groups before and at 4,24 , and 48 hours after operation

\begin{tabular}{|c|c|c|c|c|}
\hline Groups & Before & After $4 \mathrm{~h}$ & After $24 \mathrm{~h}$ & After $48 \mathrm{~h}$ \\
\hline Sham & $13.78 \pm 3.57$ & $21 \pm 4.62$ & $16.11 \pm 4.27$ & $13.89 \pm 5.38$ \\
\hline Control group & $11.44 \pm 1.22$ & $118.89 \pm 1.11^{* *}$ & $112.44 \pm 6.1^{* *}$ & $81.67 \pm 7.46 * *$ \\
\hline KhWEP 30 & $14.44 \pm 2.46$ & $84.67 \pm 12.15^{*}$ & $65.67 \pm 12.74^{*}$ & $54.11 \pm 12.36 * \#$ \\
\hline KeWEP 30 & $15.11 \pm 5.16$ & $58.22 \pm 15.24 *$ & $72.33 \pm 13.56^{*}$ & $47.67 \pm 12.83 * \#$ \\
\hline KhWEP 100 & $15.11 \pm 3.74$ & $88.44 \pm 13.03 *$ & $77.22 \pm 14.97 *$ & $64.67 \pm 12.92 * \#$ \\
\hline KeWEP 100 & $14.78 \pm 3.23$ & $76.67 \pm 12.88^{*}$ & $71.22 \pm 11.78^{*}$ & $51 \pm 17.48 * \#$ \\
\hline KhWEP 200 & $12.78 \pm 3$ & $46.67 \pm 14.38 * \dagger$ & $46.33 \pm 13.62 *+$ & $27.44 \pm 4.87 * \#+$ \\
\hline KeWEP 200 & $15.22 \pm 3.9$ & $91.89 \pm 11.64 *+\dagger$ & $62.44 \pm 14.75^{*+\dagger}$ & 65.11ะ17.43*\#†† \\
\hline
\end{tabular}

KhWEP: Propolis water extract of Khorasan Razavi province; KeWEP: Propolis water extract of Kerman province.

Data were analyzed using the two-way ANOVA, LSD post hoc test. $\mathrm{P}<0.05$ was considered as statistically significant. Data are presented as the mean $\pm \operatorname{SEM}(\mathrm{n}=9)$. $\# P<0.001$ vs. after $4 \& 24 \mathrm{~h},{ }^{* *} P<0.001$ vs. sham group, ${ }^{*} P<0.05$ vs. control group, $+P<0.05$ vs. other WEP-treated groups, $++P<0.01$ vs. KhWEP200. 
showed to be neuroprotective as assessed by decreasing the weight loss, infarct volume, brain edema and improved the neurological tests.

According to previous studies, soon after the loss of perfusion, contrary to cell death in the ischemic core, the cell in the penumbra is alive, but functionally impaired and highly at risk. If the conditions do not improve, the tissue gradually is disrupted, and the infarct core expands to the penumbra (30). A large section of morbidity and mortality from stroke is due to development of infarct core to the penumbra between the hours up to days after the initial ischemia occur (31). Unlike the core cell necrosis, apoptosis is the main event in the penumbra zone (32). Stroke triggers a cascade of complex pathological processes, resulting in spreading tissue damage and cell death to the penumbra region (4). Oxidative stress, by producing free radicals, is one of the most prominent phenomena in the pathogenesis of ischemic brain injury and can precipitate other pathological processes such as neuroinflammation and apoptosis (33). A two-fold rise in superoxide radical anion formation has been reported in the penumbra zone after focal ischemia (34). Furthermore, some studies have focused on the role of inflammation as one of the important mechanisms involved in stroke complication, particularly brain edema (31). There are few treatments for stroke; therefore, the development of novel therapeutics is necessary, notably to protect the cell penumbra (35). The basic purpose of this neuroprotective strategy is to interfere with the events of the ischemic cascade by focusing on one or more of described mechanisms and blocking the pathological processes causing the death of vulnerable nerves (36).

Variability in the constituents of propolis in different parts of the world has caused these agent to have several biological properties such as the antioxidant, anti-apoptotic, anti-inflammatory and neuroprotective activities (7,13,16,17,37-39). Although propolis is a complex mixture and synergistic interactions between its compositions probably is an essential factor in its biological activities, but many studies demonstrated that polyphenol content is responsible for these feature (3841). Propolis was shown to have robust scavenging activity in vitro towards nitric oxide and superoxide free radical $(42,43)$. Ahn and colleagues in a study on the antioxidant activity of propolis in different regions of China showed that there was a relatively strong relationship between antioxidant activity and polyphenol content (44). This substance, due to the variety of its polyphenol, especially flavonoids has also shown anti-inflammatory activity (39, 45). Propolis is not toxic, and studies have shown that a dose of $1400 \mathrm{mg} / \mathrm{kg}$ body weight per day fails to cause toxic effects in mice (39). Hence, it seems that propolis can reduce ischemic brain injury through suppressing oxidative stress and inflammation.

Kakoolaki et al reported the protective effect of Turkish propolis against neuronal cell death in fish brain through the scavenging free radicals after cypermethrin-induced neurotoxicity (19). In their research, Shimazawa and colleagues showed that green propolis from Brazil statistically decreased the infarct volume in ischemic rats (17). Our results demonstrated similar properties for IBP. According to these results, the infarct volume in animals treated with WEPs at 100 and $200 \mathrm{mg} / \mathrm{kg}$ (both regions) was significantly lower than the control group. Meanwhile, neuroinflammation is a common pathological incident reported in many different brain diseases through involvement in excitotoxicity and is usually followed by brain edema (46). Recently, Swamy et al demonstrated the neuroprotective effect of propolis in Malaysia against excitotoxicity via diminished NOS, caspase- 3 activities, nitric oxide (NO), and tumor necrosis factor alpha (TNF- $\alpha$ ) concentration involvement in neuroinflammation and apoptosis (47). In 2013, Wu et al reported that Brazilian propolis inhibited the hypoxiainduced neuroinflammatory responses in microglia (18). In this study, WEPs at doses of 30, 100 and 200 $\mathrm{mg} / \mathrm{kg}$ from both regions significantly decreased brain edema compared to control group. Reducing the infarct volume and brain edema in the IBP-treated groups could be due to the simultaneous antioxidant and antiinflammatory activity of propolis phenolic compounds and thus inhibition of apoptosis in the penumbra, that have been reported in the other areas for this substance. In this respect, quercetin and naringenin, the phenolic compounds in the KeWEP, potentially represent an antioxidative and anti-inflammatory capacity in vitro by blockade of NF- $\kappa B$ activation, inhibiting STAT-1, iNOS mRNA, and NO production in stimulated macrophages $(48,49)$. It was found that pinocembrin, a phenolic compound in the KhWEP, could protect the brain ischemic against in vitro free radicals and apoptosis. Oxidative stress and inflammation were not directly studied in this research (50).

Patients who survive from stroke, often suffer from sequelae such as neurological deficits and weight loss (29, 51). Sensorimotor function tests such as sticky tape and hanging wire are the most commonly employed tests in assessing post-stroke behavioral deficits (28). Thiyagarajan et al demonstrated that the effect of a neuroprotective agent could be evaluated through functional improvement tests regardless of considering the infarct volume (52). Results of this study also indicated that both tests followed by the induction of ischemia in mice were impaired in the control group. WEPs of both regions improved behavioral functions and decreased body weight loss. Neurologic performance improvement in functional tests and prevention of weight loss after receiving medication may be due to attenuating the progression of the ischemic lesion to the penumbra zone and reducing the infarct volume. However, more research should be conducted 
on the potential of this compound to enhance recovery of function.

The difference between similar doses of WEPs (from two regions) on the ischemic brain injury indices, measured in this study, was not statistically significant. However, better effect relative of KeWEP than KhWEP on some indices like some behavioral tests, and the dose 30 on infarct volume may be related to the higher polyphenol content as well as the difference in composition of propolis from this region that needs to be investigated, further.

\section{Conclusion}

According to our results, IBP has neuroprotective properties and reduces ischemic brain injury. These findings, together with earlier studies on propolis in other areas, would project a good hope in the endeavor to find novel stroke treatment. There are many open questions about propolis that need to be answered, and we have to do more work to increase knowledge about propolis.

\section{Acknowledgements}

The Authors would like also to thank the Clinical Research Development Unit for its support and collaboration in AliIbn Abi-Talib hospital, Rafsanjan University of Medical Sciences, Rafsanjan, Iran.

\section{Authors' contributions}

GhB, MA, AS, ZA, ZK, AV, AMA and MTB contributed to the idea of the study, its design, interpretation, preparation of the manuscript and its revise. GhB, MA, and MFN collected the Data. All read and confirmed the last version for publication.

\section{Conflict of interests}

The authors declare no conflict of interest.

\section{Ethical considerations}

All animal procedures were approved by the Research Ethics Committee of Rafsanjan University of Medical Sciences, Iran (approval code: IR.RUMC.REC.1394.165) and were in compliance with National Laws and the National Institutes of Health guidelines for the use and care of laboratory animals.

\section{Funding/Support}

This article was supported by the research vice chancellor of Mashhad, under grant No. 922335, and Rafsanjan, under grant No. 3649, Universities of Medical Sciences, Iran.

\section{References}

1. Grotta JC, Albers GW, Broderick JP, Lo EH, Kasner SE, Mendelow $\mathrm{AD}$, et al. Stroke: pathophysiology, diagnosis, and management. China: Elsevier Health Sciences; 2016. p. 234.

2. Vakilian A, Bazmandegan G, Saeedi Nezhad M, Asadollahi
Z, Moghadam Ahmadi A. Association of HbAlc Levels with Extent and Functional Status of Ischemic and Hemorrhagic Stroke. Journal of Kerman University of Medical Sciences. 2018;25(4):307-17.

3. Pirzad Jahromi G, Imani E, Nasehi M, Shahriari A. Effect of Achillea millefolium aqueous extract on memory deficit and anxiety caused by stroke in ovariectomized rats. J Herbmed Pharmacol. 2019;8(2):153-9. doi: 10.15171/ jhp.2019.24.

4. Shin WH, Park SJ, Kim EJ. Protective effect of anthocyanins in middle cerebral artery occlusion and reperfusion model of cerebral ischemia in rats. Life Sci. 2006;79(2):130-7. doi: 10.1016/j.lfs.2005.12.033.

5. Crack PJ, Taylor JM. Reactive oxygen species and the modulation of stroke. Free RadicBiolMed.2005;38(11):143344. doi: 10.1016/j.freeradbiomed.2005.01.019.

6. Wang C, Pei A, Chen J, Yu H, Sun ML, Liu CF, et al. A natural coumarin derivative esculetin offers neuroprotection on cerebral ischemia/reperfusion injury in mice. J Neurochem. 2012;121(6):1007-13. doi: 10.1111/j.14714159.2012.07744.x.

7. Bankova VS, de Castro SL, Marcucci MC. Propolis: recent advances in chemistry and plant origin. Apidologie. 2000;31(1):3-15.

8. Bazmandegan G, Boroushaki MT, Shamsizadeh A, Ayoobi F, Hakimizadeh E, Allahtavakoli M. Brown propolis attenuates cerebral ischemia-induced oxidative damage via affecting antioxidant enzyme system in mice. Biomed Pharmacother. 2017;85:503-10. doi: 10.1016/j.biopha.2016.11.057.

9. Popova M, Bankova V, Naydensky C, Tsvetkova I, Kujumgiev A. Comparative study of the biological activity of propolis from different geographic origin: a statistical approach. Macedonian Pharm Bull. 2004;50:9-14.

10. Greenaway W, May J, Scaysbrook T, Whatley FR. Identification by gas chromatography-mass spectrometry of 150 compounds in propolis. Z Naturforsch C. 1991;46(12):111-21.

11. Sutjarittangtham K, Sanpa S, Tunkasiri T, Chantawannakul P, Intatha U, Eitssayeam S. Bactericidal effects of propolis/polylactic acid (PLA) nanofibres obtained via electrospinning. J Apic Res. 2014;53(1):109-15. doi: 10.3896/IBRA.1.53.1.11.

12. Simões CC, de Araújo DB, de Castro RD, de Araújo RPC. The antimicrobial action of propolis on human saliva. J Apic Res. 2008;47(4):323-4.doi:10.1080/00218839.2008.11101483.

13. Liu R, Gao M, Yang ZH, Du GH. Pinocembrin protects rat brain against oxidation and apoptosis induced by ischemia-reperfusion both in vivo and in vitro. Brain Res. 2008;1216:104-15. doi: 10.1016/j.brainres.2008.03.049.

14. Ha SK, Moon E, Kim SY. Chrysin suppresses LPSstimulated proinflammatory responses by blocking NFkappaB and JNK activations in microglia cells. Neurosci Lett. 2010;485(3):143-7. doi: 10.1016/j.neulet.2010.08.064.

15. Kumazawa S, Hamasaka T, Nakayama T. Antioxidant activity of propolis of various geographic origins. Food Chem. 2004;84(3):329-39. doi: 10.1016/S03088146(03)00216-4.

16. Nakajima $Y$, Shimazawa $M$, Mishima $S$, Hara $H$. Neuroprotective effects of Brazilian green propolis and its main constituents against oxygen-glucose deprivation 
stress, with a gene-expression analysis. Phytother Res. 2009;23(10):1431-8. doi: 10.1002/ptr.2797.

17. Shimazawa M, Chikamatsu S, Morimoto N, Mishima S, Nagai H, Hara H. Neuroprotection by Brazilian Green Propolis against In vitro and In vivo Ischemic Neuronal Damage. Evid Based Complement Alternat Med. 2005;2(2):201-7. doi: 10.1093/ecam/neh078.

18. Wu Z, Zhu A, Takayama F, Okada R, Liu Y, Harada Y, et al. Brazilian green propolis suppresses the hypoxiainduced neuroinflammatory responses by inhibiting NFkappaB activation in microglia. Oxid Med Cell Longev. 2013;2013:906726. doi: 10.1155/2013/906726.

19. Kakoolaki S, Talas ZS, Cakir O, Ciftci O, Ozdemir I. Role of propolis on oxidative stress in fish brain. Basic Clin Neurosci. 2013;4(2):153-8.

20. Daleprane JB, Freitas Vda S, Pacheco A, Rudnicki M, Faine LA, Dorr FA, et al. Anti-atherogenic and anti-angiogenic activities of polyphenols from propolis. J Nutr Biochem. 2012;23(6):557-66. doi: 10.1016/j.jnutbio.2011.02.012.

21. Najafi MF, Vahedy F, Seyyedin M, Jomehzadeh HR, Bozary K. Effect of the water extracts of propolis on stimulation and inhibition of different cells. Cytotechnology. 2007;54(1):4956. doi: 10.1007/s10616-007-9067-2.

22. Mohammadzadeh S, Sharriatpanahi M, Hamedi M, Amanzadeh Y, Sadat Ebrahimi SE, Ostad SN. Antioxidant power of Iranian propolis extract. Food Chem. 2007;103(3):729-33. doi: 10.1016/j.foodchem.2006.09.014.

23. Dizaji A, Valizadeh E, Alishah H, Shaddel A, Sis N, Shiran $\mathrm{M}$, et al. Chemical composition analysis and antimicrobial activity of Iranian propolis. Res J Biol Sci. 2008;3(5):448-50.

24. Yap P, Abu Bakar MF, Lim H, Carrier D. Antibacterial activity of polyphenol-rich extract of selected wild honey collected in Sabah, Malaysia. J Apic Res. 2015;54(3):163-72. doi: 10.1080/00218839.2016.1151633.

25. Vakili A. Mini-review for methods of induction experimental ischemia stroke: type of model, evaluation ischemic damage and neurological deficit. Koomesh. 2006;8(1):1-10. [Persian].

26. Allahtavakoli M, Amin F, Esmaeeli-Nadimi A, Shamsizadeh A, Kazemi-Arababadi M, Kennedy D. Ascorbic Acid Reduces the Adverse Effects of Delayed Administration of Tissue Plasminogen Activator in a Rat Stroke Model. Basic Clin Pharmacol Toxicol. 2015;117(5):335-9. doi: 10.1111/ bcpt.12413.

27. Bouet V, Boulouard M, Toutain J, Divoux D, Bernaudin $\mathrm{M}$, Schumann-Bard P, et al. The adhesive removal test: a sensitive method to assess sensorimotor deficits in mice. Nat Protoc. 2009;4(10):1560-4. doi: 10.1038/nprot.2009.125.

28. Balkaya M, Krober JM, Rex A, Endres M. Assessing poststroke behavior in mouse models of focal ischemia. J Cereb Blood Flow Metab. 2013;33(3):330-8. doi: 10.1038/ jcbfm.2012.185.

29. Tahamtan M, Allahtavakoli M, Abbasnejad M, Roohbakhsh A, Taghipour Z, Taghavi M, et al. Exercise preconditioning improves behavioral functions following transient cerebral ischemia induced by 4 -vessel occlusion (4-VO) in rats. Arch Iran Med. 2013;16(12):697-704. doi: 0131612/aim.004.

30. Ramos-Cabrer P, Campos F, Sobrino T, Castillo J. Targeting the ischemic penumbra. Stroke. 2011;42(1 Suppl):S7-11. doi: $10.1161 /$ strokeaha.110.596684.
31. Dirnagl U, Iadecola C, Moskowitz MA. Pathobiology of ischaemic stroke: an integrated view. Trends Neurosci. 1999;22(9):391-7. doi: 10.1016/s0166-2236(99)01401-0.

32. Khan M, Elango C, Ansari MA, Singh I, Singh AK. Caffeic acid phenethyl ester reduces neurovascular inflammation and protects rat brain following transient focal cerebral ischemia. J Neurochem. 2007;102(2):365-77. doi: 10.1111/j.1471-4159.2007.04526.x.

33. Heo JH, Han SW, Lee SK. Free radicals as triggers of brain edema formation after stroke. Free Radic Biol Med. 2005;39(1):51-70. doi: 10.1016/j. freeradbiomed.2005.03.035.

34. Cherubini A, Ruggiero C, Polidori MC, Mecocci P. Potential markers of oxidative stress in stroke. Free Radic Biol Med. 2005;39(7):841-52. doi: 10.1016/j. freeradbiomed.2005.06.025.

35. Doyle KP, Simon RP, Stenzel-Poore MP. Mechanisms of ischemic brain damage. Neuropharmacology. 2008;55(3):310-8. doi: 10.1016/j.neuropharm.2008.01.005.

36. Auriel E, Bornstein NM. Neuroprotection in acute ischemic stroke--current status. J Cell Mol Med. 2010;14(9):2200-2. doi: 10.1111/j.1582-4934.2010.01135.x.

37. Barbosa RA, Nunes TL, Nunes TL, da Paixão AO, Belo Neto R, Moura S, et al. Hydroalcoholic extract of red propolis promotes functional recovery and axon repair after sciatic nerve injury in rats. Pharm Biol. 2016;54(6):993-1004. doi: 10.3109/13880209.2015.1091844.

38. Zhang J, Shen X, Wang K, Cao X, Zhang C, Zheng H, et al. Antioxidant activities and molecular mechanisms of the ethanol extracts of Baccharis propolis and Eucalyptus propolis in RAW64.7 cells. Pharm Biol. 2016;54(10):222035. doi: 10.3109/13880209.2016.1151444.

39. Victorino FR, Franco SL, Estivalet Svidzinski TI, AvilaCampos MJ, Nakamura Cuman RK, Hidalgo MM, et al. Pharmacological evaluation of propolis solutions for endodontic use. Pharm Biol. 2007;45(9):721-7. doi: $10.1080 / 13880200701575411$.

40. de Almeida EC, Menezes H. Anti-inflammatory activity of propolis extracts: a review. J Venom Anim Toxins. 2002;8(2):191-212. doi: 10.1590/S010479302002000200002 .

41. Machado BA, Silva RP, Barreto Gde A, Costa SS, Silva DF, Brandão HN, et al. Chemical Composition and Biological Activity of Extracts Obtained by Supercritical Extraction and Ethanolic Extraction of Brown, Green and Red Propolis Derived from Different Geographic Regions in Brazil. PLoS One. 2016;11(1):e0145954. doi: 10.1371/journal. pone. 0145954 .

42. Ichikawa H, Satoh K, Tobe T, Yasuda I, Ushio F, Matsumoto $\mathrm{K}$, et al. Free radical scavenging activity of propolis. Redox Rep. 2002;7(5):347-50. doi: 10.1179/135100002125000965.

43. Scheller S, Wilczok T, Imielski S, Krol W, Gabrys J, Shani J. Free radical scavenging by ethanol extract of propolis. Int J Radiat Biol. 1990;57(3):461-5. doi: 10.1080/09553009014552601.

44. Ahn MR, Kumazawa S, Usui Y, Nakamura J, Matsuka $\mathrm{M}$, Zhu F, et al. Antioxidant activity and constituents of propolis collected in various areas of China. Food Chem. 2007;101(4):1383-92. doi: 10.1016/j.foodchem.2006.03.045.

45. Silva JC, Rodrigues S, Feás X, Estevinho LM. Antimicrobial 
activity, phenolic profile and role in the inflammation of propolis. Food Chem Toxicol. 2012;50(5):1790-5. doi: 10.1016/j.fct.2012.02.097.

46. Fukuda AM, Badaut J. Aquaporin 4: a player in cerebral edema and neuroinflammation. J Neuroinflammation. 2012;9:279. doi: 10.1186/1742-2094-9-279.

47. Swamy M, Suhaili D, Sirajudeen KN, Mustapha Z, Govindasamy C. Propolis ameliorates tumor nerosis factoralpha, nitric oxide levels, caspase- 3 and nitric oxide synthase activities in kainic acid mediated excitotoxicity in rat brain. Afr J Tradit Complement Altern Med. 2014;11(5):48-53. doi: 10.4314/ajtcam.v11i5.8.

48. Hämäläinen $\mathrm{M}$, Nieminen $\mathrm{R}$, Vuorela $\mathrm{P}$, Heinonen $\mathrm{M}$, Moilanen E. Anti-inflammatory effects of flavonoids: genistein, kaempferol, quercetin, and daidzein inhibit STAT-1 and NF-kappaB activations, whereas flavone, isorhamnetin, naringenin, and pelargonidin inhibit only NF-kappaB activation along with their inhibitory effect on iNOS expression and NO production in activated macrophages. Mediators Inflamm. 2007;2007:45673. doi: $10.1155 / 2007 / 45673$

49. Knekt P, Isotupa S, Rissanen $H$, Heliovaara M, Jarvinen $\mathrm{R}$, Hakkinen S, et al. Quercetin intake and the incidence of cerebrovascular disease. Eur J Clin Nutr. 2000;54(5):415-7.

50. Xin BR, Liu JF, Kang J, Chan WP. (2R, 3S)-pinobanksin-3cinnamate, a new flavonone from seeds of Alpinia galanga willd., presents in vitro neuroprotective effects. Mol Cell Toxicol. 2014;10(2):165-72. doi: 10.1007/s13273-014-00187.

51. Jönsson AC, Lindgren I, Norrving B, Lindgren A. Weight loss after stroke: a population-based study from the Lund Stroke Register. Stroke. 2008;39(3):918-23. doi: 10.1161/ strokeaha.107.497602.

52. Thiyagarajan M,FernándezJA,LaneSM, GriffinJH,Zlokovic BV. Activated protein $\mathrm{C}$ promotes neovascularization and neurogenesis in postischemic brain via protease-activated receptor 1. J Neurosci. 2008;28(48):12788-97. doi: 10.1523/ jneurosci.3485-08.2008. 